\title{
WEIGHTED STATISTICAL CONVERGENCE OF REAL VALUED SEQUENCES
}

\author{
Abdu Awel Adem and Maya Altınok
}

(C) by University of Niš, Serbia | Creative Commons Licence: CC BY-NC-ND Abstract. Functions defined in the form " $g: \mathbb{N} \rightarrow[0, \infty)$ such that $\lim _{n \rightarrow \infty} g(n)=\infty$ and $\lim _{n \rightarrow \infty} \frac{n}{g(n)}=0$ " are called weight functions. Using the weight function, the concept of weighted density, which is a generalization of natural density, was defined by Balcerzak, Das, Filipczak and Swaczyna in the paper "Generalized kinsd of density and the associated ideals", Acta Mathematica Hungarica 147(1) (2015), 97-115.

In this study, the definitions of $g$-statistical convergence and $g$-statistical Cauchy sequence for any weight function $g$ are given and it is proved that these two concepts are equivalent. Also, some inclusions of the sets of all weight $g_{1}$-statistical convergent and weight $g_{2}$-statistical convergent sequences for $g_{1}, g_{2}$ which have the initial conditions are given.

Keywords: weight functions; natural density; statistical convergent sequences.

\section{Introduction}

In [5], Fast introduced the concept of statistical convergence. In [15], Schoenberg gave some basic properties of statistically convergence and also studied the concept as a summability method. After this works many Mathematician have used these concept in their studies $[8,9,10,11]$. In $[2,3]$, the authors proposed a modified version of density by replacing $n$ by $n^{\alpha}$ where $0<\alpha \leq 1$. In [1], the authors defined a more general kind of density by replacing $n^{\alpha}$ by a function $g: \mathbb{N} \rightarrow[0, \infty)$ with $\lim _{n \rightarrow \infty} g(n)=\infty$. In this paper, we will study the weighted $g$-statistically convergence concept.

Let $K$ be a subset of natural numbers. Natural density of $K$ is defined by

$$
\delta(K)=\lim _{n \rightarrow \infty} \frac{1}{n}|K(n)|
$$

where $K(n)=\{k \leq n: k \in K\}$ and the vertical bars denotes the number of elements of $K(n)$.

Received December 02, 2019; accepted February 19, 2020

2010 Mathematics Subject Classification. Primary 40A05; Secondary 46A45 
Let $g: \mathbb{N} \rightarrow[0, \infty)$ be a function with $\lim _{n \rightarrow \infty} g(n)=\infty$. Let us remember that the definition of density of weight $g(n)$.

Definition 1.1. The density of weight $g$ defined by the formula

$$
d_{g}(A)=\lim _{n \rightarrow \infty} \frac{|A(n)|}{g(n)}
$$

for $A \subset \mathbb{N}[1,4]$.

After the study [1], the concept of $g$-density was applied to various problems related to sequences and interesting results were obtained in $[4,7,12,13,14]$.

Basically in this study, it will be shown that the results given in [6] can be re-examined by using $g$-density.

In this paper, we are concerned with the subsets of natural numbers having weight $g(n)$ density zero. To facilitate this, we have introduced the following notation: If $x$ is a sequence such that $x_{k}$ satisfies property $P$ for all $k$ except a set of weight $g(n)$ density zero, then we say that $x_{k}$ satisfies $P$ for (weight $g$ almost all $k$ ) and it is denoted by $(g-a . a . k)$ for simplicity.

Definition 1.2. Let $x=\left(x_{k}\right)$ be a real valued sequence. $x$ is weight $g$-statistical convergent to the number $L$ if for each $\varepsilon>0$

$$
\lim _{n \rightarrow \infty} \frac{\left|\left\{k \leq n:\left|x_{k}-L\right| \geq \varepsilon\right\}\right|}{g(n)}=0,
$$

i.e., $\left|x_{k}-L\right|<\varepsilon \quad(g-a . a . k)$. In this case we write $g-s t-\lim x_{k}=L$.

$C_{g}^{s t}$ denotes the set of all weight $g$-statistical convergent sequences.

If we take the function $g(n)=n$ we obtain the usual statistical convergence.

It is clear that every convergent sequence is also weight $g$-statistical convergent. But the converse is not true in general.

Example 1.1. Let us define the function $g(n)=2 n$ and the sequence as

$$
x_{k}= \begin{cases}3, & k=m^{2}, \quad m \in \mathbb{N} \\ 0, & k \neq 0\end{cases}
$$

Then $\left|k \leq n: x_{k} \neq 0\right| \leq \sqrt{n}$. So, $g-s t-\lim x_{k}=0$.

Theorem 1.1. If the sequence $\left(x_{n}\right)$ is weight-g-statistical convergent to $L$ then there is a set $K=\left\{k_{1}<k_{2}<\ldots\right\}$ such that $d_{g}(K)=d_{g}(\mathbb{N})$ and $\lim _{n \rightarrow \infty} x_{k_{n}}=L$. 
Proof. Let us assume that $g-s t-l i m x_{k}=L$. Take $K_{i}:=\left\{n \in \mathbb{N}:\left|x_{n}-L\right|<\frac{1}{i}\right\}$, $(i=1,2, \ldots)$. Then by definition we have $d_{g}\left(K_{i}^{c}\right)=0$ and it is clear that $d_{g}\left(K_{i}\right)=$ $d_{g}(\mathbb{N}),(i=1,2, \ldots)$. Also it is easy to control that

$$
\ldots \subset K_{i+1} \subset K_{i} \subset \ldots \subset K_{2} \subset K_{1}
$$

Let $\left\{T_{j}\right\}_{j \in \mathbb{N}}$ be a strictly increasing sequence of positive real numbers. Let choose an arbitrary number $a_{1} \in K_{1}$. By (1.1) we can choose an element $a_{2} \in K_{2}$, $a_{2}>a_{1}$ such that for each $n \geq a_{2}$ we have $\frac{K_{2}(n)}{g(n)}>T_{2}$. Moreover choose $a_{3}>a_{2}$, $a_{3} \in K_{3}$ such that for each $n \geq a_{3}$ we have $\frac{K_{3}(n)}{g(n)}>T_{3}$. If we proceed in this way we obtain a sequence $a_{1}<a_{2} \ldots<a_{i}<\ldots$ of positive integers such that

$$
a_{i} \in K_{i},(i=1,2, \ldots) \text { and } \frac{K_{i}(n)}{g(n)}>T_{i}
$$

for each $n \geq a_{i}, i=1,2, \ldots$

Let us establish the set $K$ as follows: each natural number of the interval [1, $\left.a_{1}\right]$ belong to $K$, moreover, any natural number of the interval $\left[a_{i}, a_{i+1}\right]$ belongs to $K$ if and only if it belongs to $K_{i}(i=1,2, \ldots)$. From (1.1) and (1.2) we have

$$
\frac{K(n)}{g(n)} \geq \frac{K_{i}(n)}{g(n)}>T_{i}
$$

for each $n, a_{i} \leq n<a_{i+1}$. By last inequality it is clear that $\bar{d}_{g}(K)=\infty$.

Let $\varepsilon>0$, and choose $i$ such that $\frac{1}{i}<\varepsilon$. Let $n \geq a_{i}, n \in K$. There exists a number $t \geq i$ such that $a_{t} \leq n<a_{t+1}$. But from the definition of $K, n \in K_{t}$. Thus $\left|x_{n}-L\right|<\frac{1}{t} \leq \frac{1}{i}<\varepsilon$. Hence, $\lim _{n \rightarrow \infty} x_{k_{n}}=L$.

Remark 1.1. The converse of Theorem 1.1 is not true.

Example 1.2. Let us consider the sequence

$$
\left(x_{k}\right):= \begin{cases}1, & k=n^{2} \\ 0, & k \neq n^{2}\end{cases}
$$

and $g(n)=n^{1 / 4}$. It is clear that the set $K=\left\{k: k=n^{2}, n \in \mathbb{N}\right\} \subset \mathbb{N}$ has the property $\bar{d}_{g}(K)=\infty$. But $g-s t-\lim x_{k} \neq 1$.

Let us note that every statistical convergent sequence is also weight- $g$-statistical convergent to the same number. But the converse of this situation is not true.

Example 1.3. Let $a_{k}=2^{2^{k}}$, and

$$
g(n):=\left\{\begin{array}{cc}
a_{2 k}, & n \in\left[a_{k}, a_{k+1}\right), k=1,2, \ldots \\
1, & n<4
\end{array}\right.
$$


Let $A_{k}:=\left\{n \in \mathbb{N}: a_{k} \leq n<2 a_{k}\right\}$ and $A:=\cup_{k \geq 1} A_{k}$. Let us take account the sequence

$$
x_{n}:= \begin{cases}1, & n \in A, \\ 0, & n \notin A .\end{cases}
$$

It is clear that $\frac{1}{2} a_{k} \leq\left|A_{k}\right| \leq a_{k}$. Let us check that $x_{n} \nrightarrow 0(s t)$. If we put $m_{k}=\max A_{k}$, we obtain

$$
\frac{\left|\left\{k \leq n:\left|x_{k}-0\right| \geq \varepsilon\right\}\right|}{n}=\frac{\left|\left\{k \leq n: x_{k} \in A\right\}\right|}{n}=\frac{|A|}{m_{k}} \geq \frac{\left|A_{k}\right|}{m_{k}} \geq \frac{\frac{1}{2} a_{k}}{2 a_{k}}=\frac{1}{4}
$$

for all $k \geq 1$.

Moreover, $g-s t-\lim x_{k}=0$. For sufficiently large $\mathrm{n}$, we have

$$
\begin{aligned}
\frac{\left|\left\{k \leq n:\left|x_{k}-0\right| \geq \varepsilon\right\}\right|}{g(n)} & =\frac{\left|\left\{k \leq n: x_{k} \in A\right\}\right|}{g(n)}=\frac{|A|}{g(n)} \\
& =\frac{\left|\left\{k \leq m_{k}: x_{k} \in A\right\}\right|}{g\left(m_{k}\right)} \\
& \leq \frac{\left|A_{k}\right|}{a_{2 k}} \leq \frac{a_{k}}{a_{2 k}} \rightarrow 0 .
\end{aligned}
$$

Definition 1.3. Let $x=\left(x_{k}\right)$ be a real valued sequence. $x$ is weight $g$-statistical Cauchy sequence if for each $\varepsilon>0$ there exists a natural number $N=N(\varepsilon)$ such that

$$
\lim _{n \rightarrow \infty} \frac{\left|\left\{k \leq n:\left|x_{k}-x_{N}\right| \geq \varepsilon\right\}\right|}{g(n)}=0,
$$

i.e., $\left|x_{k}-x_{N}\right|<\varepsilon \quad(g-a . a . k)$. In this case we write $x$ is weight $g$-Cauchy sequence.

Lemma 1.1. The following statements are equivalent:

(i) $x$ is a weight $g$-statistically convergent sequence,

(ii) $x$ is a weight $g$-statistically Cauchy sequence,

(iii) $x$ is a sequence for which there is a convergent sequence $y$ such that $x_{k}=y_{k}$ $(g-a . a . k)$.

Proof. $(i) \Rightarrow($ ii) Let us assume that $x$ is a weight $g$-statistical convergent sequence. Suppose $\varepsilon>0$ and $g-s t-\lim x=L$. Then $\left|x_{k}-L\right|<\frac{\varepsilon}{2}(g-a . a . k)$ holds.

If we choose a natural number $N$ such that $\left|x_{N}-L\right|<\frac{\varepsilon}{2}$, then we have

$$
\left|x_{k}-x_{N}\right|<\left|x_{k}-L\right|+\left|x_{N}-L\right|<\frac{\varepsilon}{2}+\frac{\varepsilon}{2}(g-a . a . k) .
$$

Hence, $x$ is a weight $g$-statistical Cauchy sequence.

$($ ii $) \Rightarrow($ iii $)$ Let us assume that $x$ is a weight $g$-statistical Cauchy sequence. Choose $N(1)$ such that the interval $I=\left[x_{N(1)}-1, x_{N(1)}+1\right]$ contains $x_{k}(g-a . a . k)$. Also apply (ii) to choose $M$ such that $I^{\prime}=\left[x_{M}-\frac{1}{2}, x_{M}+\frac{1}{2}\right]$ contains $x_{k}(g-a . a . k)$. We claim that

$$
I_{1}=I \cap I^{\prime} \text { contains } x_{k}(g-a . a . k),
$$


for

$$
\left\{k \leq n: x_{k} \notin I \cap I^{\prime}\right\}=\left\{k \leq n: x_{k} \notin I\right\} \cup\left\{k \leq n: x_{k} \notin I^{\prime}\right\} .
$$

Thus,

$$
\begin{aligned}
& \lim _{n \rightarrow \infty} \frac{1}{g(n)}\left|\left\{k \leq n: x_{k} \notin I \cap I^{\prime}\right\}\right| \leq \\
\leq & \lim _{n \rightarrow \infty} \frac{1}{g(n)}\left|\left\{k \leq n: x_{k} \notin I\right\}\right|+\lim _{n \rightarrow \infty} \frac{1}{g(n)}\left|\left\{k \leq n: x_{k} \notin I^{\prime}\right\}\right|=0 .
\end{aligned}
$$

So, $I_{1}$ is closed interval of length less than or equal to 1 and contains $x_{k}(g-a . a . k)$. Now we continue by choosing $N(2)$ such that $I^{\prime \prime}=\left[x_{N(2)}-\frac{1}{4}, x_{N(2)}+\frac{1}{4}\right]$ contains $x_{k}(g-a . a . k)$, by the previously argument $I_{2}=I_{1} \cap I^{\prime \prime}$ contains $x_{k}(g-a . a . k)$, and $I_{2}$ has length less than or equal to $\frac{1}{2}$. Proceeding inductively we construct a sequence $\left\{I_{m}\right\}_{m=1}^{\infty}$ of closed intervals such that for each $m, I_{m+1} \subseteq I_{m}$, and the length of $I_{m}$ is not greater than $2^{1-m}$, and $x_{k} \in I_{m}(g-a . a . k)$. From the Nested Interval Theorem there is a number $\alpha$ such that $\alpha=\cap_{m=1}^{\infty} I_{m}$. If we use $x_{k} \in I_{m}(g-a . a . k)$, we can choose an increasing positive sequence $\left\{T_{m}\right\}_{m=1}^{\infty}$ such that

$$
\frac{1}{g(n)}\left|\left\{k \leq n: x_{k} \notin I_{m}\right\}\right|<\frac{1}{g(m)} \text { if } n>T_{m} .
$$

Next define a subsequence $z$ of $x$ consisting of all terms $x_{k}$ such that $k>T_{1}$ and if $T_{m}<k \leq T_{m+1}$ then $x_{k} \notin I_{m}$.

Now define the sequence $y$ by

$$
y_{k}=\left\{\begin{aligned}
\alpha, & \text { if } x_{k} \text { is a term of } z \\
x_{k}, & \text { otherwise. }
\end{aligned}\right.
$$

Then $\lim y_{k}=\alpha$; for, if $\varepsilon>\frac{1}{g(m)}>0$ and $k>T_{m}$ then either $x_{k}$ is a term of $z$, which means $y_{k}=\alpha$ or $y_{k}=x_{k} \in I_{m}$ and $\left|y_{k}-\alpha\right| \leq$ length of $I_{m}<2^{1-m}$. We also assert that $x_{k}=y_{k}(g-a . a . k)$. To confirm this we observe that if $T_{m}<n<T_{m+1}$ then

$$
\left\{k \leq n: y_{k} \neq x_{k}\right\} \subseteq\left\{k \leq n: x_{k} \notin I_{m}\right\}
$$

so from $(1.3)$

$$
\frac{1}{g(n)}\left|\left\{k \leq n: y_{k} \neq x_{k}\right\}\right| \leq \frac{1}{g(n)}\left|\left\{k \leq n: x_{k} \notin I_{m}\right\}\right|<\frac{1}{g(m)}
$$

is obtained. Thus, the limit as $n \rightarrow \infty$ is 0 and $x_{k}=y_{k}(g-a . a . k)$.

$($ iii $) \Rightarrow(i)$ Let us assume that $x_{k}=y_{k}(g-a . a . k)$ and $\lim y_{k}=L$. Suppose $\varepsilon>0$. Then for each $n$,

$$
\left\{k \leq n:\left|x_{k}-L\right|>\varepsilon\right\} \subseteq\left\{k \leq n: x_{k} \neq y_{k}\right\} \cup\left\{k \leq n:\left|y_{k}-L\right|>\varepsilon\right\}
$$


from the assumption $\lim y_{k}=L$, the second set contains a fixed number of integers, say $l=l(\varepsilon)$. So,

$$
\begin{aligned}
\lim _{n \rightarrow \infty} \frac{1}{g(n)}\left|\left\{k \leq n:\left|x_{k}-L\right|>\varepsilon\right\}\right| & \leq \lim _{n \rightarrow \infty} \frac{1}{g(n)}\left|\left\{k \leq n: x_{k} \neq y_{k}\right\}\right|+ \\
& +\lim _{n \rightarrow \infty} \frac{l}{g(n)}=0
\end{aligned}
$$

because $x_{k}=y_{k}(g-a . a . k)$. Hence, $\left|x_{k}-L\right| \leq \varepsilon(g-a . a . k)$. So, the proof is complete.

Corollary 1.1. Let $x$ be a real valued sequence. If $g-s t-\lim x_{k}=L$, then $x$ has a subsequence $y$ such that $\lim y_{k}=L$.

\section{Inclusion Between Two $g-s t$-Convergence}

Let $G$ denotes the set of all functions $g: \mathbb{N} \rightarrow[0, \infty)$ satisfying the condition $g(n) \rightarrow \infty$ and $\frac{n}{g(n)} \nrightarrow 0$. In this section, we will introduce some inclusions between various $g \in G$.

Lemma 2.1. Let $g_{1}, g_{2} \in G$ such that there exist $M, m>0$ and $k_{0} \in \mathbb{N}$ such that $m \leq \frac{g_{1}(n)}{g_{2}(n)} \leq M$ for all $n \geq k_{0}$. Then $C_{g_{1}}^{s t}(x)=C_{g_{2}}^{s t}(x)$.

Proof. Suppose the sequence $x$ is weight $g_{1}$-statistical convergence to $L$. This implies that for each $\varepsilon>0$

$$
\lim _{n \rightarrow \infty} \frac{\left|\left\{k \leq n:\left|x_{k}-L\right| \geq \varepsilon\right\}\right|}{g_{1}(n)}=0 .
$$

Together with the fact that $\frac{g_{1}(n)}{g_{2}(n)} \leq M$, this implies that

$$
\frac{\left|\left\{k \leq n:\left|x_{k}-L\right| \geq \varepsilon\right\}\right|}{M g_{2}(n)} \leq \frac{\left|\left\{k \leq n:\left|x_{k}-L\right| \geq \varepsilon\right\}\right|}{g_{1}(n)} .
$$

for all $n \geq k_{0}$. This implies

$$
\lim _{n \rightarrow \infty} \frac{\left|\left\{k \leq n:\left|x_{k}-L\right| \geq \varepsilon\right\}\right|}{M g_{2}(n)} \leq \lim _{n \rightarrow \infty} \frac{\left|\left\{k \leq n:\left|x_{k}-L\right| \geq \varepsilon\right\}\right|}{g_{1}(n)}=0 .
$$

From the hypothesis we obtain

$$
\lim _{n \rightarrow \infty} \frac{\left|\left\{k \leq n:\left|x_{k}-L\right| \geq \varepsilon\right\}\right|}{g_{2}(n)}=0 .
$$

Thus, the sequence $x$ is weight $g_{2}$-statistical convergent to $L$. So, $C_{g_{1}}^{s t}(x) \subset C_{g_{2}}^{s t}(x)$. We can prove the iclusion $C_{g_{2}}^{s t}(x) \subset C_{g_{1}}^{s t}(x)$ by similar way. 
Lemma 2.2. For each function $f \in G$ there exists a nondecreasing function $g \in G$ such that $C_{f}^{s t}(x)=C_{g}^{s t}(x)$. Moreover,

$$
g(n) \leq f(n)
$$

for all $n \in \mathbb{N}$.

Proof. If $f$ is nondecreasing, it is nclear. Otherwise, define the related function $g: \mathbb{N} \rightarrow[0, \infty)$ as follows. Let $a_{1}=\min \{f(n): n \in \mathbb{N}\}, i_{1}=\max \left\{i \in \mathbb{N}: f(i)=a_{1}\right\}$ and $g(i)=a_{1}$ for $0 \leq i \leq i_{1}$. Next, let $a_{2}=\min \left\{f(n): n>i_{1}\right\}, i_{2}=\max \{i \in N$ : $\left.f(i)=a_{2}\right\}$ and $g(i)=a_{2}$ for $i_{1}<i \leq i_{2}$. Rest of the function $g$ is established by induction.

Obviously, the function $g$ is nondecreasing and $g(n) \rightarrow \infty$. By the construction, $g(n) \leq f(n)$, for all $n \in \mathbb{N}$. Hence $\frac{n}{f(n)} \leq \frac{n}{g(n)}$ for all $n$ which implies that $\frac{n}{g(n)} \nrightarrow 0$. Thus $g \in G$.

Let $\left(x_{n}\right)$ be a weight $g$-statistical convergent sequence to $L$. So, for each $\varepsilon>0$

$$
\lim _{n \rightarrow \infty} \frac{\left|\left\{k \leq n:\left|x_{k}-L\right| \geq \varepsilon\right\}\right|}{g(n)}=0
$$

holds. From (2.1) we have following inequality

$$
\frac{\left|\left\{k \leq n:\left|x_{k}-L\right| \geq \varepsilon\right\}\right|}{f(n)} \leq \frac{\left|\left\{k \leq n:\left|x_{k}-L\right| \geq \varepsilon\right\}\right|}{g(n)} .
$$

If we take limit when $n \rightarrow \infty$ we obtain $f-s t-\lim x_{k}=L$. Thus, the inclusion $C_{g}^{s t} \subset C_{f}^{s t}$.

By construction, for each $n \in \mathbb{N}$ there exist $m \geq n$ such that $g(n)=g(m)=$ $f(m)$. Suppose that $x_{n} \nrightarrow L(g-s t)$. Then there exists $a$, where $a \in \mathbb{R}^{+} \cup\{+\infty\}$ and an inreasing sequence $\left(n_{i}\right)$ of indices such that

$$
\lim _{i \rightarrow \infty} \frac{\left|\left\{k \leq n_{i}:\left|x_{k}-L\right| \geq \varepsilon\right\}\right|}{g\left(n_{i}\right)}=a>0 .
$$

For each $i \in \mathbb{N}$ we can find $m_{i} \geq n_{i}$ such that $g\left(n_{i}\right)=g\left(m_{i}\right)=f\left(m_{i}\right)$. Hence

$$
\frac{\left|\left\{k \leq n_{i}:\left|x_{k}-L\right| \geq \varepsilon\right\}\right|}{g\left(n_{i}\right)} \leq \frac{\left|\left\{k \leq m_{i}:\left|x_{k}-L\right| \geq \varepsilon\right\}\right|}{f\left(m_{i}\right)}
$$

holds. So, $x_{n} \nrightarrow L(f-s t)$.

Lemma 2.3. Let $f \in G$ be such that $\frac{n}{f(n)} \rightarrow \infty, L, \varepsilon$ real numbers with $\varepsilon>0$. Then there exists a sequence $\left(x_{n}\right)$ such that $\left(\frac{\left|\left\{k \leq n:\left|x_{k}-L\right| \geq \varepsilon\right\}\right|}{f(n)}\right)$ is bounded but not convergent to zero. 
Proof. Firstly, let us assume that $f$ is nondecreasing. Take to the smallest non negative integer, $k_{0}$, such that for $n \geq k_{0}, f(n)>2$. Let us define a set $A \subset$ $\mathbb{N} \backslash\left\{0,1,2, \ldots k_{0}-1\right\}$ inductively, deciding whether $n \geq k_{0}$ should belong to $A$ or not. Let $n \notin A$ for all $n<k_{0}$. Suppose that $n \geq k_{0}$ and then we have defined $A(n)$. If $\frac{|A(n)|}{f(n+1)}<1$ then let $n \in A$. Otherwise, let $n \notin A$. So, we construct the set $A$. From this construction and the condition $f(n) \rightarrow \infty, A$ is infinite.

We assert that $\mathbb{N} \backslash A$ is also infinite. Let us assume that it is finite and choose $n_{0} \in \mathbb{N}$ such that $n \in A$ for all $n \geq n_{0}$. Then, we have

$$
\frac{n-n_{0}}{f(n+1)} \leq \frac{|A(n)|}{f(n+1)}<1
$$

for all $n \geq n_{0}$. But this is impossible because of the assumption, $\frac{n-n_{0}}{f(n+1)} \rightarrow \infty$. Now, we will show that $\frac{|A(n)|}{f(n)}<2$ for each $n \geq k_{0}$. It is clear that if $n=k_{0}$ it is true. Suppose that $\frac{|A(n)|}{f(n)}<2$ for a fixed $n \geq k_{0}$.

If $\frac{|A(n)|}{f(n+1)}<1$, we have

$$
\begin{aligned}
\frac{|A(n+1)|}{f(n+1)} & =\frac{|A(n)|}{f(n+1)}+\frac{1}{f(n+1)} \\
& \leq \frac{|A(n)|}{f(n+1)}+\frac{1}{f(n)} \\
& \leq 1+\frac{1}{2}<2 .
\end{aligned}
$$

If $\frac{|A(n)|}{f(n+1)}>1$, then $n \notin A$ and so,

$$
\frac{|A(n+1)|}{f(n+1)}=\frac{|A(n)|}{f(n+1)} \leq \frac{|A(n)|}{f(n)}<2 .
$$

Now, let us define a sequence $\left(x_{n}\right)$ as follows:

$$
x_{n}:=\left\{\begin{array}{cc}
n & n \in A \\
L & n \notin A
\end{array}\right.
$$

where $L \in \mathbb{R}$ is a fixed number. It is clear that the sequence $\left(\frac{\left|\left\{k \leq n:\left|x_{k}-L\right| \geq \varepsilon\right\}\right|}{f(n)}\right)$ is bounded from the first part of this proof.

Now, we will show that the sequence $\left(\frac{\left|\left\{k \leq n:\left|x_{k}-L\right| \geq \varepsilon\right\}\right|}{f(n)}\right)$ is not convergent to 0 . For this aim consider any $n \geq k_{0}$. We will find $m \geq n$ such that $\frac{|A(m)|}{f(m)} \geq 1$. If $\frac{|A(n)|}{f(n)} \geq 1$, put $m:=n$. Otherwise, choose the smallest $m \geq n$ such that $m \in \mathbb{N} \backslash A$. Then $\frac{|A(m)|}{f(m+1)} \geq 1$ and so, $\frac{|A(m)|}{f(m)} \geq 1$. Thus, the sequence $\left(\frac{\left|\left\{k \leq n:\left|x_{k}-L\right| \geq \varepsilon\right\}\right|}{f(n)}\right)$ is not convergent to 0 . 
Now, let us back to the general case where $f \in G$ need not be nondecreasing. Then we assume the associated function $g \in G$ from Lemma 2.2. Note that $\frac{n}{g(n)} \rightarrow$ $\infty$ since $\frac{n}{g(n)} \geq \frac{n}{f(n)}$ for all $n$ and $\frac{n}{f(n)} \rightarrow \infty$. By the above reasons we obtain the respective set $A$ for $g$. Thus, $\frac{|A(n)|}{g(n)} \nrightarrow 0$ and the sequence $\left(\frac{|A(n)|}{g(n)}\right)$ is bounded. Then $\frac{|A(n)|}{f(n)} \nrightarrow 0$, and the sequence $\left(\frac{\left|\left\{k \leq n:\left|x_{k}-L\right| \geq \varepsilon\right\}\right|}{f(n)}\right)$ is bounded since $g(n) \leq f(n)$ for all $n \in \mathbb{N}$.

Theorem 2.1. If $g_{1}, g_{2}$ belong to $G$ such that $\frac{g_{2}(n)}{g_{1}(n)} \rightarrow \infty$ then $C_{g_{1}}^{s t}(x) \subsetneq C_{g_{2}}^{s t}(x)$. If $g \in G$ and $\frac{n}{g(n)} \rightarrow \infty$ then $C_{g}^{s t}(x) \subsetneq C^{s t}(x)$.

Proof. To prove the first claim note that the inclusion $C_{g_{1}}^{s t}(x) \subset C_{g_{2}}^{s t}(x)$ follows from Lemma 2.1. Set $f:=\sqrt{g_{1} \cdot g_{2}}$. Then

$$
\lim _{n \rightarrow \infty} \frac{f(n)}{g_{1}(n)}=\lim _{n \rightarrow \infty} \frac{g_{2}(n)}{f(n)}=\infty
$$

Also we have

$$
\frac{n}{g_{1}(n)}=\frac{n}{g_{2}(n)} \cdot \frac{g_{2}(n)}{g_{1}(n)} \rightarrow \infty
$$

So $\frac{n}{f(n)}=\sqrt{\frac{n^{2}}{g_{1}(n) g_{2}(n)}} \rightarrow \infty$. Hence $f$ have the assumption of Lemma 2.3. Take the sequence $\left(x_{n}\right)$ obtained in this lemma. Then $x_{n} \in C_{g_{2}}^{s t}(x)$ but $x_{n} \notin C_{g_{1}}^{s t}(x)$. Indeed, using (2.2) we have

$$
\frac{\left|\left\{k \leq n:\left|x_{k}-L\right| \geq \varepsilon\right\}\right|}{g_{2}(n)}=\frac{\left|\left\{k \leq n:\left|x_{k}-L\right| \geq \varepsilon\right\}\right|}{f(n)} \cdot \frac{f(n)}{g_{2}(n)} \rightarrow 0
$$

because $\left(\frac{\left|\left\{k \leq n:\left|x_{k}-L\right| \geq \varepsilon\right\}\right|}{f(n)}\right)_{n \in \mathbb{N}}$ is bounded from Lemma 2.3. Thus, $x_{n} \in C_{g_{2}}^{s t}(x)$. To prove that $x_{n} \notin C_{g_{1}}^{s t}(x)$ observe that

$$
\frac{\left|\left\{k \leq n:\left|x_{k}-L\right| \geq \varepsilon\right\}\right|}{g_{1}(n)}=\frac{\left|\left\{k \leq n:\left|x_{k}-L\right| \geq \varepsilon\right\}\right|}{f(n)} \frac{f(n)}{g_{1}(n)} .
$$

So, $x_{n} \notin C_{g_{1}}^{s t}(x)$ because $\frac{\left|\left\{k \leq n:\left|x_{k}-L\right| \geq \varepsilon\right\}\right|}{f(n)} \nrightarrow 0$, and $\frac{f(n)}{g_{1}(n)} \rightarrow \infty$ from $(2.2)$.

If we take $g_{2}(n)=n$, for all $n \in \mathbb{N}$, second assertion proved easily from the same way.

Corollary 2.1. Let $0<\alpha<\beta \leq 1$ and $g_{1}(n)=n^{\alpha}, g_{2}=n^{\beta}$ for $n \in \mathbb{N}$. Then $C_{g_{1}}^{s t}(x) \subsetneq C_{g_{2}}^{s t}(x)$.

Example 2.1. Let

$$
g_{1}(n)=\left\{\begin{aligned}
n, & \text { for even } n \in \mathbb{N} \\
\sqrt{n}, & \text { for odd } n \in \mathbb{N}
\end{aligned}\right.
$$


and $g_{2}(n)=\sqrt{n}$ for $n \in \mathbb{N}$. It is clear that, $\lim _{\sup _{n \rightarrow \infty}} \frac{g_{1}(n)}{g_{2}(n)}=\infty$. However, $C_{g_{1}}^{s t}(x)=$ $C_{g_{2}}^{s t}(x)$. Indeed, construct a nondecreasing function $g \in G$ such that $C_{g}^{s t}(x)=C_{g_{1}}^{s t}(x)$, according to the method used in the proof of Lemma 2.1. Then it follows from simple calculations that $g$ is given by

$$
g(n)=\left\{\begin{aligned}
\sqrt{n+1} & \text { for even } n \in \mathbb{N} \\
\sqrt{n} & \text { for odd } n \in \mathbb{N} .
\end{aligned}\right.
$$

Obviously, $\frac{1}{2} \leq \frac{g(n)}{g_{2}(n)} \leq 2$ for all $n \geq 1$. Therefore, by Lemma 2.1 we have $C_{g}^{s t}(x)=C_{g_{1}}^{s t}(x)$.

Theorem 2.2. There exists a function $g \in G$ such that $C_{g}^{s t}$ is different from $C_{n^{\alpha}}^{s t}$ with $0<\alpha<1$.

Proof. Let $a_{k}$ and $g(n)$ defined as in Example 1.3. Let $A_{k}:=\left\{n \in \mathbb{N}: a_{k+1}-\right.$ $\left.\left(a_{k+1}\right)^{1 / 4} \leq n<a_{k+1}\right\}$ and $A=\cup_{k \geq 2} A_{k}$. Let us take account the sequence

$$
x_{n}=\left\{\begin{array}{cc}
n, & n \in A \\
0, & n \notin A .
\end{array}\right.
$$

It is clear that $\frac{1}{2}\left(a_{k+1}\right)^{1 / 4} \leq\left|B_{k}\right| \leq\left(a_{k+1}\right)^{1 / 4}$. Let us check that $g-s t-\lim x_{k} \neq$ 0 . For $k>0$ we have

$$
\frac{\left|\left\{k \leq a_{k+1}-1:\left|x_{k}-0\right| \geq \varepsilon\right\}\right|}{g\left(a_{k+1}-1\right)} \geq \frac{\frac{1}{2}\left|B_{k}\right|}{g\left(a_{k}\right)} \geq \frac{\frac{1}{4}\left(a_{k+1}\right)^{1 / 4}}{\left(a_{k+1}\right)^{1 / 4}}=\frac{1}{4},
$$

so, $g-s t-\lim x_{k} \neq 0$. Furthermore,

$$
\left|\left\{k \leq a_{k+1}:\left|x_{k}-0\right| \geq \varepsilon\right\}\right| \leq\left(a_{k}\right)^{1 / 4}+\left(a_{k+1}\right)^{1 / 4} \leq 2\left(a_{k+1}\right)^{1 / 4}
$$

and so,

$$
\frac{\left|\left\{k \leq a_{k+1}:\left|x_{k}-0\right| \geq \varepsilon\right\}\right|}{\left(a_{k+1}\right)^{1 / 3}} \leq \frac{2\left(a_{k+1}\right)^{1 / 4}}{\left(a_{k+1}\right)^{1 / 3}}=2\left(a_{k+1}\right)^{-1 / 12} \rightarrow 0, \quad(k \rightarrow \infty)
$$

holds.

Now, fix any $n \geq 4$ and choose a unique $k \in \mathbb{N}$ such that $n \in\left[a_{k}, a_{k+1}\right)$. If $n<a_{k+1}-\left(a_{k+1}\right)^{1 / 4}$ then

$$
\begin{aligned}
\frac{\left|\left\{k \leq n:\left|x_{k}-0\right| \geq \varepsilon\right\}\right|}{n^{1 / 3}} & =\frac{\left|\left\{k \leq a_{k}:\left|x_{k}-0\right| \geq \varepsilon\right\}\right|}{n^{1 / 3}} \\
& \leq \frac{\left|\left\{k \leq n:\left|x_{k}-0\right| \geq \varepsilon\right\}\right|}{\left(a_{k}\right)^{1 / 3}} \leq 2\left(a_{k}\right)^{-1 / 12} .
\end{aligned}
$$

If $a_{k+1}-\left(a_{k+1}\right)^{1 / 4} \leq n<a_{k+1}$ then for $b>a>0$, the function

$$
f(x):=\frac{a+x}{(b+x)^{1 / 3}}, x \geq 0
$$


is increasing, thus

$$
\frac{\left|\left\{k \leq n:\left|x_{k}-0\right| \geq \varepsilon\right\}\right|}{n^{1 / 3}} \leq \frac{\left|\left\{k \leq a_{k+1}:\left|x_{k}-0\right| \geq \varepsilon\right\}\right|}{\left(a_{k+1}\right)^{1 / 3}} .
$$

So, $x_{n} \in C_{n^{1 / 3}}^{s t}(x)$.

Now, let $0<\alpha<1, \alpha \neq \frac{1}{3}$. If $\alpha<\frac{1}{3}$ then from Corollary $2.1 C_{n^{\alpha}}^{s t} \subsetneq C_{n^{1 / 3}}^{s t}$ and $C_{g}^{s t} \backslash C_{n^{\alpha}}^{s t} \neq \varnothing$ because $C_{g}^{s t} \backslash C_{n^{1 / 3}}^{s t} \neq \varnothing$. If $\alpha>\frac{1}{3}$ then $C_{n^{\alpha}}^{s t} \backslash C_{g}^{s t} \neq \varnothing$. By the same way we can show that $x_{n} \in C^{s t} \backslash C_{g}^{s t}$. So $C_{g}^{s t} \subsetneq C^{s t}$.

\section{Acknowledgement}

The authors would like to thank Professor Mehmet Küçükaslan for his discussions some steps during the preparation of this paper.

\section{R E F E R E N C E S}

1. M. Balcerzak, P. Das, M. Filipczak and J. Swaczyna: Generalized kinds of density and the associated ideals. Acta Math. Hungar. 147(1) (2015), 97-115.

2. S. Bhunia, P. DAS and S. K. PAL: Restricting statistical convergence. Acta Mathematica Hungarica, 134(1-2) (2012), 153-161.

3. R. ÇOLAK: Statistical convergence of order $\alpha$. Modern Methods in Analysis and Its Applications, Anamaya Pub., New Delhi, India (2010), 121-129.

4. P. DAs and E. SAVAŞ: On generalized statistical and ideal convergence of metric-valued sequences. Reprinted in Ukrainian Math. J. 68(12) (2017), 1849-1859. Ukrain. Mat. Zh. 68(12) (2016), 1598-1606.

5. H. FAST: Sur la convergence statistique. Colloq. Math. 2 (1951), 241-244.

6. J. A. Fridy: On statistical convergence. Analysis 5 (1985), 301-313.

7. Ş. KoncA, M. KÜÇÜKASLAN and E. GENÇ: I-statistical convergence of double sequences defined by weight functions in a locally solid Riesz space. Konuralp J. Math. 7(1) (2019), 55-61.

8. M. KÜÇüKaslan and M. YILmaztüRK: On deferred statistical convergence of sequences. Kyungpook Math. J., 56 (2006), 357-366.

9. M. Yilmaztürk, O. Mizrak and M. KüÇüKaslan: Deferred statistical cluster points of real valued sequences. Univ. J. Appl. Math., 1 (2013), 1-6.

10. E. SAVAŞ: On some generalizd sequence spaces defined by modulus. Indian J. Pure Appl. Math., 30(5) (1999), 973-978.

11. E. SAvAŞ: Strong almost convergence and almost $\lambda$-statistical convergence. Hokkaido Math., 29(3) (2000), 531-536.

12. E. SAVAS and P. DAS: On I-statistical and I-lacunary statistical convergence of weight g. Bull. Math. Anal. Appl., 11(2) (2019), 2-11.

13. E. SAvaş: On I-lacunary statistical convergence of weight $g$ of sequences of sets. Filomat 31(16) (2017), 5315-5322. 
14. E. SAVAŞ: $I_{\theta}$-statistical convergence of weight $g$ in topological groups. Mathematics and computing, Springer Proc. Math. Stat., 253, Springer, Singapore, 2018, 43-51.

15. I. J. Schoenberg: The integrability of certain functions and related summability methods. The American Mathematical Monthly 66(5) (1959), 361-775.

Abdu Awel Adem

Mersin University

Faculty of Science and Arts

Department of Mathematics

33343 Mersin, Turkey

abdua90@gmail.com

Maya Altınok

Tarsus University

Faculty of Technology

Department of Natural and Mathematical Sciences

33460 Mersin, Turkey

mayakantar@gmail.com or mayaaltinok@tarsus.edu.tr 